\title{
ON NONINVERTIBLE LINKS WITH INVERTIBLE PROPER SUBLINKS
}

\author{
W. C. WHITTEN, JR.
}

Abstract. The title supports the primary objective of this paper. Specifically, for each $\mu \geqq 3$, a noninvertible, oriented, ordered link of $\mu$ components tamely imbedded in $S^{3}$ is exhibited with the property that each proper sublink is invertible. The case $\mu=2$ has been covered in a previous paper.

In 1963, H. F. Trotter gave a solution to a difficult problem of classical knot theory by exhibiting a collection of noninvertible knots [8]. More recently examples of noninvertible links were given [9], each link consisting of two components each of which is invertible. An oriented, ordered link $L$ of $\mu$ components in the oriented 3-sphere $S$ is invertible if there is an orientation-preserving autohomeomorphism of $S$ taking each component of $L$ on to itself with reversal of orientation. In this paper we solve a "Brunnian type" problem on invertibility of links by presenting for each $\mu \geqq 3$ a noninvertible link of $\mu$ components with the feature that each proper sublink is invertible. Such a link is shown in Figure 1.

I wish to thank W. R. Alford and K. Murasugi for conversations. I would like also to thank the referee for a helpful comment concerning notation.

Remark. The link $L$ of Figure 1 is assumed to have $\mu \geqq 3$ components. Note that each component is of trivial knot type in $S$, and that each proper sublink of $L$ is invertible. We now proceed to prove that $L$ itself, however, is noninvertible.

Let $V$ denote a closed solid torus tamely imbedded in $S-K_{\mu}$. The core of $V$ is to be of knot type $5_{1}$. If $V^{\prime}$ is a tubular neighborhood of the component $K_{\mu}^{\prime}$ of the link $L^{\prime}$ of Figure 2 and if the closure of $V^{\prime}$ does not otherwise meet $L^{\prime}$, then we make the further requirement of $V$ that there be a homeomorphism of the closure of the complement of $V^{\prime}$ in $S$ onto $V$ which takes $K_{\alpha}^{\prime}$ on to $K_{\alpha}(\alpha=1, \cdots, \mu-1)$. A section of $V$ is indicated by the heavy black lines in the lower righthand portion of Figure 1.

Now let $\lambda$ denote the core of $V$ and set $\Lambda=K_{\mu} \cup \lambda$. Orient $\lambda$ as shown in Figure 3. We have

Received by the editors January 6, 1970 .

AMS 1970 subject classifications. Primary 55A25; Secondary 57A10.

Key words and phrases. Classical knot theory, noninvertible knots, noninvertible links, "Brunnian type" invertibility problem. 


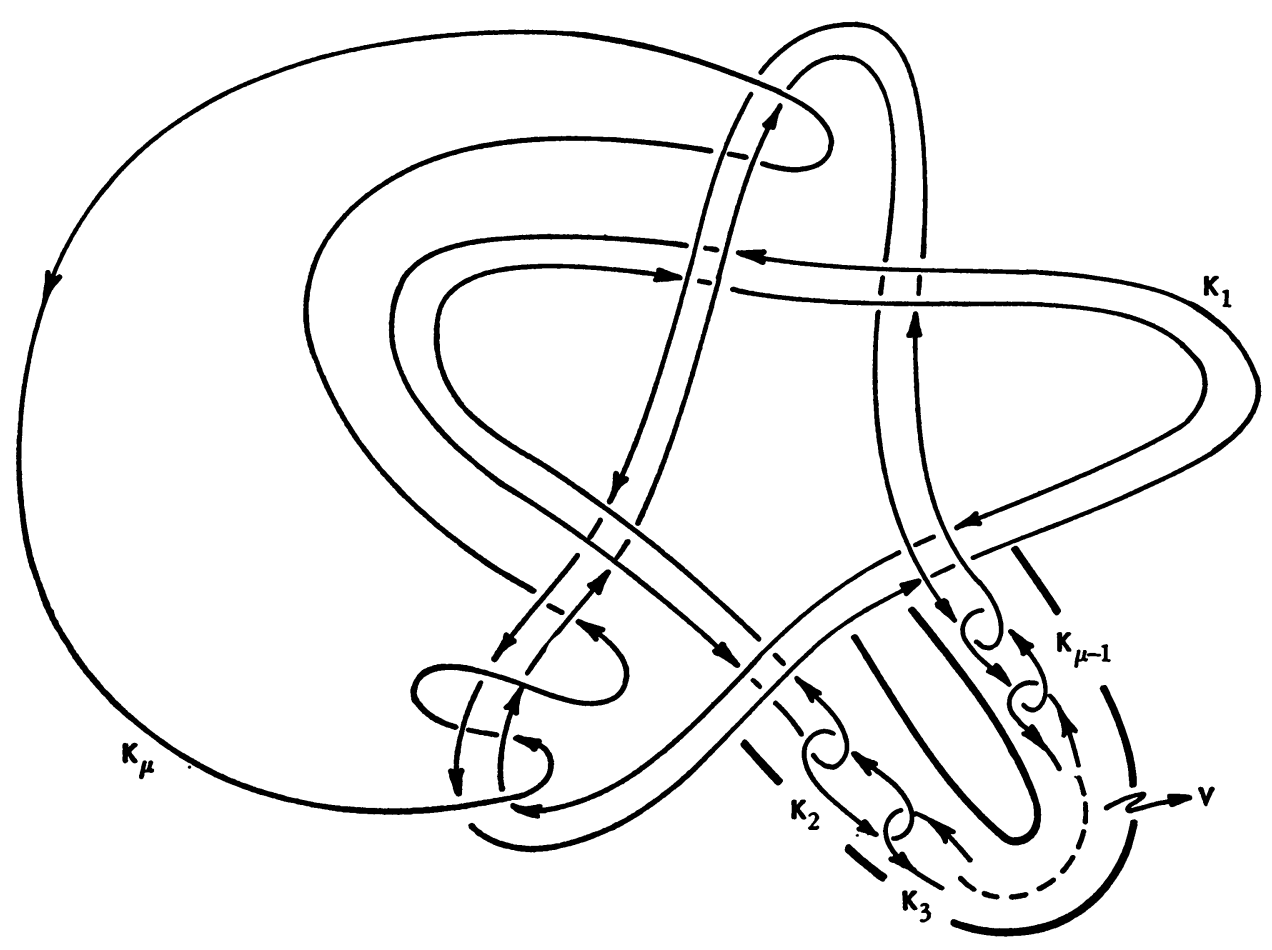

Figure 1

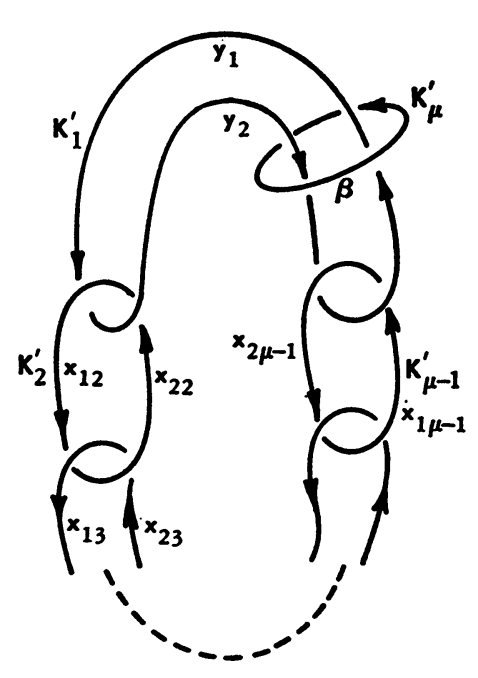

FIGURE 2

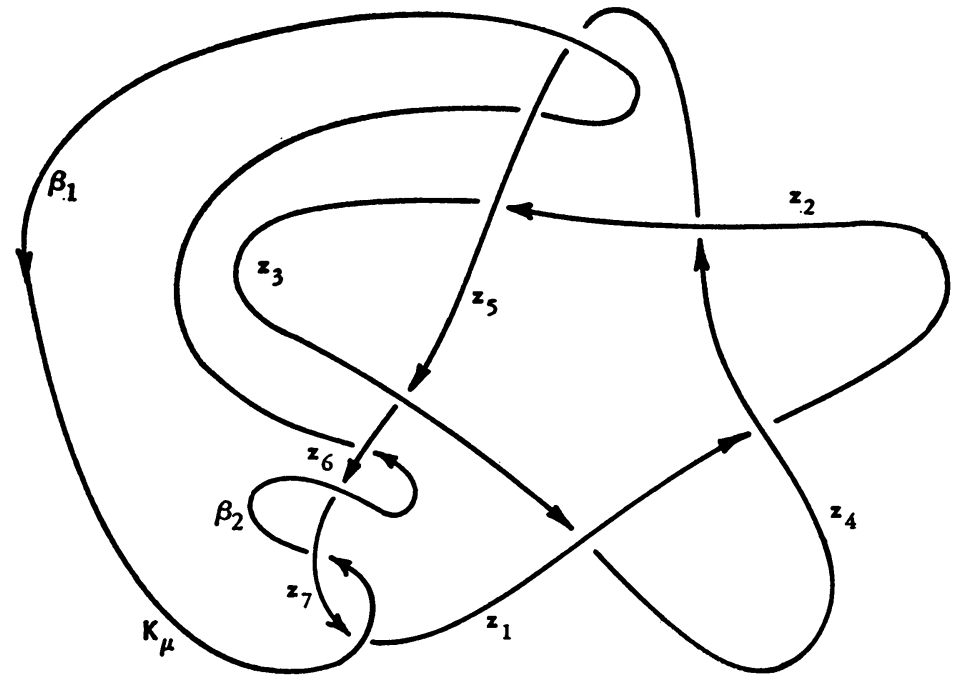

FIGURE 3 


$$
\begin{aligned}
\pi_{1}\left(S-\left(K_{\mu} \cup \operatorname{Int} V\right)\right) \approx & \pi_{1}(S-\Lambda) \\
=\mid & \beta_{1}, \beta_{2}, z_{1}, \cdots, z_{7}: \beta_{1} z_{7} \beta_{2}^{-1} z_{7}^{-1}, \beta_{2} z_{6} z_{5}^{-1} \beta_{1}^{-1} z_{5} z_{6}^{-1}, \\
& z_{1} z_{4} z_{2}^{-1} z_{4}^{-1}, z_{2} z_{5} z_{3}^{-1} z_{5}^{-1}, z_{3} z_{1} z_{4}^{-1} z_{1}^{-1}, \\
& z_{4} z_{2} \beta_{1}^{-1} z_{5}^{-1} \beta_{1} z_{2}^{-1}, z_{5} z_{3} z_{6}^{-1} z_{3}^{-1}, z_{6} \beta_{2} z_{7}^{-1} \beta_{2}^{-1}, z_{7} \beta_{1} z_{1}^{-1} \beta_{1}^{-1} \mid
\end{aligned}
$$

The group $\pi_{1}\left(V-L^{*}\right)$, where $L^{*}=K_{1} \cup \cdots \cup K_{\mu-1}$, is isomorphic to the group of the link in Figure 2, so that

$$
\begin{aligned}
\pi_{1}\left(V-L^{*}\right) \approx & \beta, x_{12}, x_{22}, \cdots, x_{1 \mu-1}, x_{2 \mu-1}, y_{1}, y_{2}: y_{1} x_{12}^{-1} y_{2}^{-1} x_{12}, \\
& y_{2} x_{22} y_{2}^{-1} x_{12}^{-1}, x_{12} x_{13}^{-1} x_{22}^{-1} x_{13}, x_{22} x_{23} x_{22}^{-1} x_{13}^{-1}, \cdots, \\
& x_{1 \mu-2} x_{1 \mu-1}^{-1} x_{2 \mu-2}^{-1} x_{1 \mu-1}, x_{2 \mu-2} x_{2 \mu-1} x_{2 \mu-2}^{-1} x_{1 \mu-1}^{-1}, \\
& x_{1 \mu-1} \beta^{-1} y_{1}^{-1} \beta x_{2 \mu-1}^{-1} \beta^{-1} y_{1} \beta, x_{2 \mu-1} \beta^{-1} y_{2} \beta x_{2 \mu-1}^{-1} \beta^{-1} y_{1}^{-1} \beta, \\
& \beta\left(y_{1}^{-1} y_{2}\right) \beta^{-1}\left(y_{1}^{-1} y_{2}\right)^{-1} \mid .
\end{aligned}
$$

For the link $K_{1}^{\prime} \cup \ldots \cup K_{\mu-1}^{\prime}$, which is a proper sublink of that in Figure 2, we have

$$
\begin{array}{r}
\pi_{1}\left(S-K_{1}^{\prime} \cup \cdots \cup K_{\mu-1}^{\prime}\right)=\mid y_{1}, y_{2}, x_{12}, x_{22}, \cdots, x_{1 \mu-1}, \\
x_{2 \mu-1}: y_{1} x_{12}^{-1} y_{2}^{-1} x_{12}, y_{2} x_{22} y_{2}^{-1} x_{12}^{-1}, x_{12} x_{13}^{-1} x_{22}^{-1} x_{13}, \\
x_{22} x_{23} x_{22}^{-1} x_{13}^{-1}, x_{13} x_{14}^{-1} x_{23}^{-1} x_{14}, x_{23} x_{24} x_{23}^{-1} x_{14}^{-1}, \cdots, \\
x_{1 \mu-2} x_{1 \mu-1}^{-1} x_{2 \mu-2}^{-1} x_{1 \mu-1}, x_{2 \mu-2} x_{2 \mu-1} x_{2 \mu-2}^{-1} x_{1 \mu-1}^{-1}, \\
x_{1 \mu-1} y_{1}^{-1} x_{2 \mu-1}^{-1} y_{1}, x_{2 \mu-1} y_{2} x_{2 \mu-1}^{-1} y_{1}^{-1} \mid .
\end{array}
$$

In order to prove that $\pi_{1}(S-L)$ is the free product of a certain pair of groups with amalgamated subgroup (see the theorem below), we need the intuitively obvious fact contained in the following

LEMma. The element $y_{1}^{-1} y_{2}$ of the group $\pi_{1}\left(S-K_{1}^{\prime} \cup \ldots \cup K_{\mu-1}^{\prime}\right)$ of (3) is not the identity.

PROOF. For convenience let $\pi_{1}\left(S-K_{1}^{\prime} \cup \ldots \cup K_{\mu-1}^{\prime}\right)$ be denoted by $D_{\mu}$. Then

$$
\begin{aligned}
D_{3}= & \mid Y_{1}, Y_{2}, X_{12}, X_{22}: Y_{1} X_{12}^{-1} Y_{2}^{-1} X_{12}, Y_{2} X_{22} Y_{2}^{-1} X_{12}^{-1}, \\
& X_{12} Y_{1}^{-1} X_{22}^{-1} Y_{1}, X_{22} Y_{2} X_{22}^{-1} Y_{1}^{-1} \mid \\
= & \left|Y_{1}, X_{12}: X_{12} Y_{1}^{-1} X_{12} Y_{1}^{-1}=Y_{1}^{-1} X_{12} Y_{1}^{-1} X_{12}\right| .
\end{aligned}
$$


Since it is assumed that $\mu \geqq 3$ always, there is a rather natural homomorphism of $\pi_{1}\left(S-K_{1}^{\prime} \cup \ldots \cup K_{\mu-1}^{\prime}\right)$ on to $D_{3}$ given by

$$
\begin{aligned}
& y_{1}, x_{13}, x_{15}, \cdots, x_{1 \mu-2} \rightarrow Y_{1} \\
& y_{2}, x_{23}, x_{25}, \cdots, x_{2 \mu-2} \rightarrow Y_{2} \\
& x_{12}, x_{14}, x_{16}, \cdots, x_{1 \mu-1} \rightarrow X_{12} \\
& x_{22}, x_{24}, x_{26}, \cdots, x_{2 \mu-1} \rightarrow X_{22},
\end{aligned}
$$

provided $\mu$ is odd. Thus, if $\mu$ is odd and $y_{1}^{-1} y_{2}=1$ in

$$
\pi_{1}\left(S-K_{1}^{\prime} \cup \ldots \cup K_{\mu-1}^{\prime}\right),
$$

then $D_{3} \approx D_{3} /\left\langle Y_{1}^{-1} Y_{2}\right\rangle$. ( $\left(\left\langle Y_{1}^{-1} Y_{2}\right\rangle\right.$ is the consequence in $D_{3}$ of $Y_{1}^{-1} Y_{2}$.) But the Alexander polynomial of $D_{3}$ is $X-Y$ and that of $D_{3} /\left\langle Y_{1}^{-1} Y_{2}\right\rangle$ is 1 ; (this latter group is free abelian of rank two).

Now if $\mu$ is even then, similar to above, we have a homomorphism of $D_{\mu}$ onto $D_{4}$, or $D_{6}$, or $D_{8}$ according as $\mu-1$ is of the form $3+6 n$, or $5+6 n$, or $7+6 n$, respectively, where $n$ is a nonnegative integer. To conclude the proof of the lemma, we need only show that $D_{k} \not D_{k} /\left\langle Y_{1}^{-1} Y_{2}\right\rangle,(k=4,6,8)$. But straightforward and easy calculations reveal that the Alexander ideal (see p. 209 of [2]) of $D_{k} /\left\langle Y_{1}^{-1} Y_{2}\right\rangle$ for each of $k=4,6,8$ is not principal. This concludes the proof of the lemma.

THEOREM. The group $\pi_{1}(S-L)$ of the link $L=K_{1} \cup \ldots \cup K_{\mu}$ of Figure 1 is isomorphic to the free product of the groups $\pi_{1}\left(S-\left(K_{\mu} \cup \operatorname{Int} V\right)\right)$ and $\pi_{1}\left(V-L^{*}\right)$ amalgamated with respect to $\pi_{1}(\partial V)$, where $L^{*}$ denotes the proper sublink $K_{1} \cup \ldots \cup K_{\mu-1}$ of $L$.

Proof. Choose a point $x$ on $\partial V$ as common basepoint for the four groups. Taking $x$ also as the basepoint of the group $\pi_{1}(S-\operatorname{Int} V)$, it follows from [6] and [1] that the inclusion homomorphism $\pi_{1}(\partial V) \rightarrow \pi_{1}(S-$ Int $V)$ is a monomorphism, since $V$ is knotted in $S$; hence, $a$ fortiori, the inclusion homomorphism $\pi_{1}(\partial V) \rightarrow \pi_{1}\left(S-\left(K_{\mu} \cup\right.\right.$ Int $\left.\left.V\right)\right)$ is a monomorphism.

From (2) and the definition of $V$ it is clear that an element of $\pi_{1}(\partial V)$ can be written in the form $\beta^{r}\left(y_{1}^{-1} y_{2}\right)^{s}$, where $r$ and $s$ are integers. Killing $\beta$ in (2) maps $\pi_{1}\left(V-L^{*}\right)$ homomorphically on to the group $\pi_{1}\left(S-K_{1}^{\prime} \cup \ldots \cup K_{\mu-1}^{\prime}\right)$, (see Figure 2), and sends $\beta^{r}\left(y_{1}^{-1} y_{2}\right)^{s}$ onto $\left(y^{-1} y_{2}\right)^{s}$. If $\beta^{r}\left(y_{1}^{-1} y_{2}\right)^{s}=1$ in $\pi_{1}\left(V-L^{*}\right)$, then $\left(y_{1}^{-1} y_{2}\right)^{s}=1$ in $\pi_{1}\left(S-K_{1}^{\prime} \cup \ldots \cup K_{\mu-1}^{\prime}\right)$. Since, by the lemma, $y_{1}^{-1} y_{2} \neq 1$ in this latter group, it follows from Corollary (31.9), p. 23, of [6] that $s=0$. Hence, $\beta^{r}\left(y_{1}^{-1} y_{2}\right)^{s}=1$ in $\pi_{1}\left(V-L^{*}\right)$ implies that $\beta^{r}=1$. Since $\beta \neq 1$ in $\pi_{1}\left(V-L^{*}\right)$ and since $\pi_{1}\left(V-L^{*}\right)$ is the group of a link in $S$, it follows, again from 
[6], that $r=0$. Thus, the inclusion $\partial V \rightarrow V-L^{*}$ induces a monomorphism $\pi_{1}(\partial V) \rightarrow \pi_{1}\left(V-L^{*}\right)$.

The theorem follows by an application of van Kampen's theorem [5].

From the theorem it follows immediately that the inclusion map $S-\left(K_{\mu} \cup \operatorname{Int} V\right) \rightarrow S-L$ induces a monomorphic imbedding of $\pi_{1}\left(S-\left(K_{\mu} \cup\right.\right.$ Int $\left.\left.V\right)\right)$ in $\pi_{1}(S-L)$. As common basepoint for these two groups choose a point $x$ on $\partial V$.

We consider the covering space $\Sigma$ of $S-\left(K_{1} \cup \ldots \cup K_{\mu-1}\right)$ branched over $K_{\mu}$ whose associated unbranched covering space of $S-L$ arises from the conjugacy class of subgroups of $\pi_{1}(S-L)$ to which $\pi_{1}\left(S-\left(K_{\mu} \cup\right.\right.$ Int $\left.V\right)$ ) belongs (see [3] or $\$ 8$ of [4]). If $\Xi$ denotes the set of points in $\Sigma$ lying over $K_{\mu}$, then (1) gives a presentation for $\pi_{1}(\Sigma-\Xi)$. From the geometric interpretation of the generators of this presentation (see Figures 1 and 3), it is clear that a presentation for $\pi_{1}(\Sigma)$ is obtained from (1) simply by killing $\beta_{1}$ and $\beta_{2}$. Consequently,

$$
\pi_{1}(\Sigma) \approx\left|z_{1}, z_{4}: z_{1} z_{4} z_{1} z_{4} z_{1}=z_{4} z_{1} z_{4} z_{1} z_{4}\right| .
$$

Now the element $w=z_{7} z_{6} z_{5}^{-1}$ in $\pi_{1}\left(S-\left(K_{\mu} \cup \operatorname{Int} V\right)\right) \subset \pi_{1}(S-L)$ is clearly a longitude of the component $+K_{\mu}$ of $L$ (see Figures 1 and 3). Thus, any orientation-preserving autohomeomorphism $\psi$ of $S$ inverting $L$ induces an au tomorphism of $\pi_{1}(S-L)$ taking $w$ on to a conjugate of its inverse. In fact we can so choose a path from the basepoint $x$ to its image $\psi(x)$ that the automorphism induced by $\psi$ takes $w$ onto $w^{-1}$. This means then that the automorphism of $\pi_{1}(\Sigma)$, induced by the autohomeomorphism of $\Sigma$ (determined up to a covering translation) which in turn is induced by $\psi$, can be assumed to carry the element

$$
v=z_{1}^{2}\left(z_{1} z_{4}\right) z_{1}^{-1}\left(z_{1} z_{4}\right)^{-1}
$$

of $\pi_{1}(\Sigma)$ onto its inverse. $v$ is the element of $\pi_{1}(\Sigma)$ represented by a path in $\Sigma-\Xi$ representing $w$ in $\pi_{1}(\Sigma-\Xi)$. The groups $\pi_{1}(\Sigma-\Xi)$ and $\pi_{1}(\Sigma)$ are assumed to have the same basepoint in $\Sigma-\Xi$, this common basepoint lying over $x$ in $\partial V$.

Under the assumption then that $L$ is invertible, it follows that

(5) There is an automorphism $\phi$ of $\pi_{1}(\Sigma)$ with the property that $\phi(v)=v^{-1}$.

We shall prove that (5) is not true.

Referring to (4), it is seen that

$$
\pi_{1}(\Sigma) \approx\left|\sigma, \omega: \sigma^{2}=\omega^{5}\right|
$$


where $z_{1}=\omega^{-2} \sigma, z_{4}=\sigma^{-1} \omega^{3}$. By the work of Schreier [7] each automorphism of this group can be written in the form

$$
\sigma \rightarrow \gamma \sigma^{\epsilon} \gamma^{-1}, \quad \omega \rightarrow \gamma \omega^{\star} \gamma^{-1} \quad(\epsilon= \pm 1),
$$

for some $\gamma$ in $\pi_{1}(\Sigma)$. Writing $v$ in terms of $\sigma$ and $\omega$ we obtain

$$
v=\left(\omega^{-2} \sigma\right)^{3} \sigma^{-1} \omega^{3} \sigma^{-1} \omega,
$$

and it follows from (5) and what has been said that

(6) There is an element $\gamma$ in $\pi_{1}(\Sigma)$ such that either

(a) $\gamma\left[\left(\omega^{-2} \sigma\right)^{3} \sigma^{-1} \omega^{3} \sigma^{-1} \omega\right] \gamma^{-1}=\omega^{-1} \sigma \omega^{-3} \sigma\left(\sigma^{-1} \omega^{2}\right)^{3}$, or

(b) $\gamma\left[\left(\omega^{2} \sigma^{-1}\right)^{3} \sigma \omega^{-3} \sigma \omega^{-1}\right] \gamma^{-1}=\omega^{-1} \sigma \omega^{-3} \sigma\left(\sigma^{-1} \omega^{2}\right)^{3}$.

It was shown, however, in [9] that (6b) cannot hold. A similar argument shows that (6a) also cannot hold, and we conclude that the link $L$ of Figure 1 is noninvertible.

\section{REFERENCES}

1. M. Dehn, Über die Topologie des drei-dimensionalen Raumes, Math. Ann. 69 (1910), 137-168.

2. R. H. Fox, Free differential calculus. II: The isomorphism problem of groups, Ann. of Math. (2) 59 (1954), 196-210. MR 15, 931.

3. - Covering spaces with singularities, Algebraic geometry and topology, A symposium in honor of S. Lefschetz, Princeton Univ. Press, Princeton, N.J., 1957, pp. 243-257. MR 23 \#A626.

4. - A quick trip through knot theory, Topology of 3-Manifolds and Related Topics (Proc. Univ. of Georgia Inst., 1961), Prentice-Hall, Englewood Cliffs, N. J., 1962, pp. 120-167. MR 25 \#3522.

5. E. R. van Kampen, On the connection between the fundamental groups of some related spaces, Amer. J. Math. 55 (1933), 261-267.

6. C. D. Papakyriakopoulos, On Dehn's lemma and the asphericity of knots, Ann. of Math. (2) 66 (1957), 1-26. MR 19, 761.

7. O. Schreier, Über die Gruppen $A^{a} B^{b}=1$, Abh. Math. Sem. Univ. Hamburg 3 (1924), 167-169.

8. H. F. Trotter, Non-invertible knots exist, Topology 2 (1964), 275-280. MR 28 \#1618; errata 30, p. 1203.

9. W. C. Whitten, Jr., A pair of non-invertible links, Duke Math. J. 36 (1969), 695-698.

University OF SOUThWESTERn LOUISIANA, LAFAyetTe, Louisiana 70501 